\title{
Partial differential feedback: An on-line training configuration for the optimization of multidimensional cue learning*
}

\author{
PAUL J. HOFFMAN \\ Oregon Research Institute \\ University of Oregon, Eugene, Oregon 97403
}

The present paper describes a PDP-9 computer configuration arranged for experimental research into the acquisition of judgmental skill, but designed to overcome certain deficiences noted in stimulus-response-outcome (SRO) paradigms. The configuration departs radically from traditional approaches in that (a) the stimulus is placed under $S$ control; (b) the requirement for a "response" is eliminated; (c) outcome feedback is a continuously changing function of the multiattribute stimulus settings under the control of $S$; and (d) the configuration makes possible the rapid learning of an essential feature of such learning tasks, namely the partial derivatives of the criterion or outcome variable with respect to each of the stimulus attributes.

In this paper, we will be describing an on-line training procedure by means of which Ss may be capable of learning rather complex concepts much more efficiently than has heretofore been possible. The concern of the paper is that of the learning of multidimensional judgment concepts. These learning tasks have in the past been studied by means of the familiar paradigm in which a stimulus is presented on a given trial, a response is elicited from $S$, and this is followed by some type of reinforcement, knowledge of results, or similar outcome feedback, under the control of $E$. The stimulus varies from trial to trial in more than one dimension, i.e., it is multiattribute.

The foregoing is often referred to as the SRO (stimulus-response-outcome) paradigm for learning under controlled feedback conditions. The $\mathrm{E}$ predetermines an exact functional relationship between the stimulus attribute values and the desired outcome for all possible problems in the problem space. The focus of the experiment is on S's acquisition of knowledge about the "true" functional relationship between stimulus attribute values and criterion. Changes in performance over time signify learning effects, and these may be identified with characteristics of the attributes of the stimuli to the nature of the outcome feedback provided or to other factors implicit or explicit in the experimental design.

To represent the task in a formal notation scheme, consider an s-dimensional stimulus, whose values are displayed to $\mathrm{S}$ with instructions to respond along a given response dimension. $Y$.

*This study was supported by Grant MH 12972 from the National Institute of Mental Health and by RR05612 from the National Institutes of Heaith, United States Public Health Service.
For the $i^{\text {th }}$ trial, in the sequence $(i=1,2, \ldots, n)$, we represent the $j^{\text {th }}$ attribute value of the stimulus as $X_{i j}$ $(j=1,2, \ldots, s)$.

Then the outcome rule is a relation predetermined by $E$, which can in general be expressed as

$$
\hat{\mathrm{Y}}_{\mathrm{i}}=\mathrm{F}\left(\mathrm{X}_{\mathrm{ij}}, \theta_{\mathrm{k}}\right) \quad(\mathrm{k}=1,2, \ldots, \mathrm{t})
$$

where the $\theta_{\mathrm{k}}$ are parameters of the function. The training problem is that of determining the conditions which optimize the acquisition of judgmental skill such that, for some later sequence, $u_{1}, u_{2}, \ldots, u_{v}$

$$
\delta=\sum_{u=1}^{\mathrm{v}} \mathrm{G}\left(\mathrm{Y}_{\mathrm{u}}-\hat{\mathrm{Y}}_{\mathrm{u}}\right)=\min
$$

As $\delta \rightarrow 0$, it can be said that $S$ "learns" the functions $\mathrm{F}(\mathrm{X}, \theta)$, or that he "understands" the concept.

The purpose of the present paper is to suggest an alternative to this paradigm; one which exploits the capability of a dedicated computer and which promises to extend significantly the capabilities of $\mathrm{Ss}$ in the learning of such concepts. Before doing so, however, it is helpful for an understanding of multidimensional concept learning to look at the laboratory training techniques that were used a dozen years ago as a basis for training. This will provide an interesting contrast with the on-line experimental paradigm which is now being proposed.

\section{TRADITIONAL OUTCOME FEEDBACK (SRO)}

The normal acquisition paradigm is modeled after classical reinforcement-theoretic considerations. The task is considered to be made up of a series of sequences,

$$
S \rightarrow R \rightarrow O
$$

indicating that (a) the stimulus, $X_{\mathbf{i}}$, is displayed, (b) the response, $Y_{i}$, is evoked, and (c) the outcome feedback, $\hat{\mathrm{Y}}_{\mathrm{i}}$, is provided. One excellent SRO study that illustrates the use of laboratory equipment in the learning of abstract dimensional concepts is that of Uhl (1960). In this study. Ss acquired dimensional judgment concepts by evaluating the worth of each of a series of stimulus cards: the correct value was a linear function of the amounts of three "attributes" shown on the card. Figure 1 shows one of the stimulus cards used by Uhl. Here, each of the rows represents one of the attribute dimensions of the stimulus. and the location of a circle in the row represents its value. A series of such cards was 


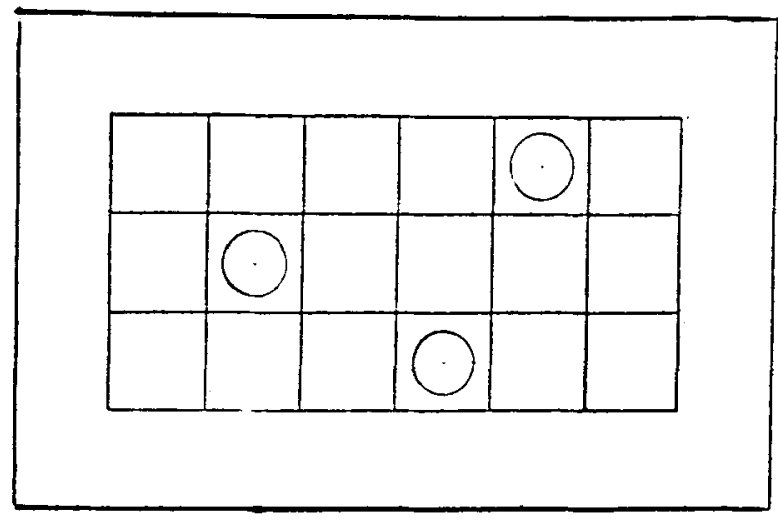

Fig. 1. A sample stimulus card (from Uhl, 1960).

displayed, and at each presentation $S$ was required to estimate the card's "value." Thus, in one task, the value increased in steps of $\$ 12.00$ along the upper row (from $\$ 0.00$ to $\$ 60.00)$; in steps of $\$ 4.50$ along the middle row (from $\$ 0.00$ to $\$ 22.50$ ): and in $\$ 7.50$ steps along the bottom row (from $\$ 0.00$ to $\$ 37.50$ ). If the columns of the card are assigned integer values $1,2, \ldots, 6 \mathrm{from}$ left to right, the value of the $i^{\text {th }}$ card can be expressed as

$$
\hat{\mathbf{Y}}_{\mathrm{i}}=\mathbf{F}\left(\mathbf{X}_{\mathrm{ij}}, \theta_{\mathbf{k}}\right)=\theta_{0}+\theta_{1} \mathrm{X}_{\mathrm{i} 1}+\theta_{2} \mathbf{X}_{\mathrm{i} 2}+\theta_{3} \mathbf{X}_{\mathrm{i} 3}
$$

for which, in this case,

$$
\begin{aligned}
& \theta_{0}=-24.00 \\
& \theta_{1}=12.00 \\
& \theta_{2}=4.50 \\
& \theta_{3}=7.50
\end{aligned}
$$

The experimental apparatus is shown in Fig. 2. At each trial, a card was displayed, $S$ recorded his estimate of the value, and this was followed by a display of the correct, or computed, value in the aperture.

By now, of course, such training studies can be easily engineered for on-line application. What is required is that the $S$ be in interaction with a computer capable of controlling the experimental sequence, generating the necessary stimulus displays, and recording the responses. Good illustrations of these techniques for multiple-cue probability learning are found in Casiellan (1972) and Hammond and Boyle (1971). The latter have described a system with graphics capability provided by a CDC-282 CRT console on line to a CDC. 6400 by means of which they and their co-workers have investigated a number of situations requiring responses along ordered scales.

The range of studies conducted under SRO learning conditions amply demonstrates the ability of Ss to learn a wide variety of dimensional concept tasks. However, considerable inefficiencies are inherent in such learning paradigms, although this seems not to have been generally acknowledged. The present author has presented an analysis of the SRO task. which highlights a number of reasons for inefficient learning. 1 These include: (1) random arrangement of sequences favors habit interference rather than efficient learning: (2) randomness in the stimuli makes difficult the perception of relations among the attributes or between the attributes and the desired response; (3) the relationship between stimulus and feedback is such as to provide only the most limited information concerning the underlying lawfulness, so that the task does not favor the perception of the given relationship by the $S$.

The remainder of this paper presents a reconceptualization of the training paradigm. An on-line training procedure is described in which the PDP-9 computer facilitates the learning of multidimensional concepts. The reconceptualization has both power and simplicity in its application to multidimensional concept learning.

\section{PARTIAL DIFFERENTIAL FEEDBACK (PDF)}

Consistent with the previous formal definition of the task, we assume an $F(X, \theta)$ that is continuously differentiable with respect to each of its parameters, $\theta$. As will shortly be shown, our objective is to restructure the task so as to optimize the acquisition of knowledge of the partial derivatives $\partial \hat{Y} / \partial X_{j}$ in the attribute-criterion space.

The stimulus display consists of a $20 \times 30 \mathrm{~cm}$ metal chassis with five vertical slots, each permitting a plastic knob of $1.5-\mathrm{cm}$ diam to traverse a distance of $15.0 \mathrm{~cm}$. The location of each knoh represents the value of an attribute, and the momentary location of all knobs represents a configuration of attribute values, descriptive of the problem. For ease of reference, each of the slots was scaled in units ranging from 0 to 100 .

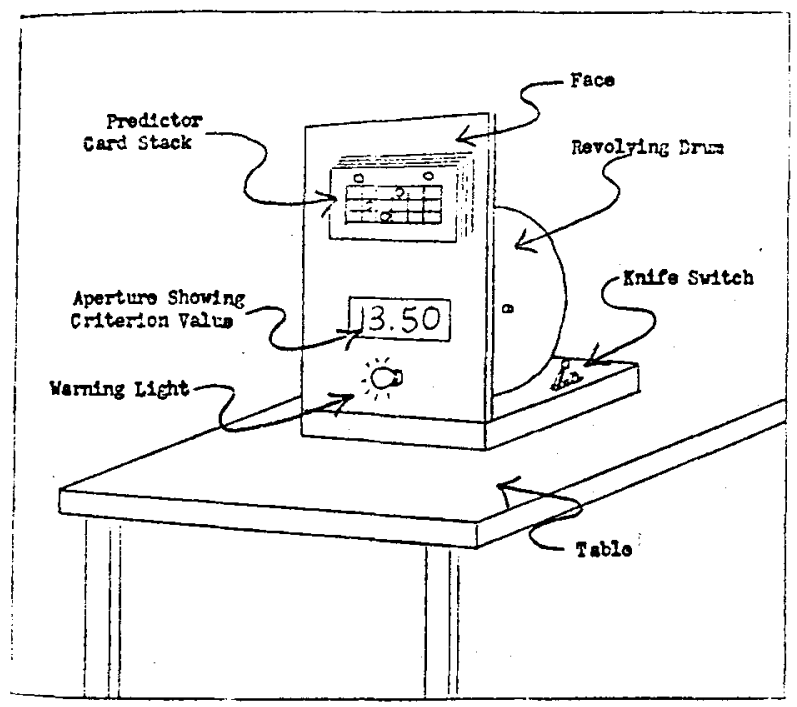

Fig. 2. A sketch of the display apparatus used by Uhl (1960). Ss were seated directly in front of the face and could not see the revolving drum (with forthcoming criterion values attached). 


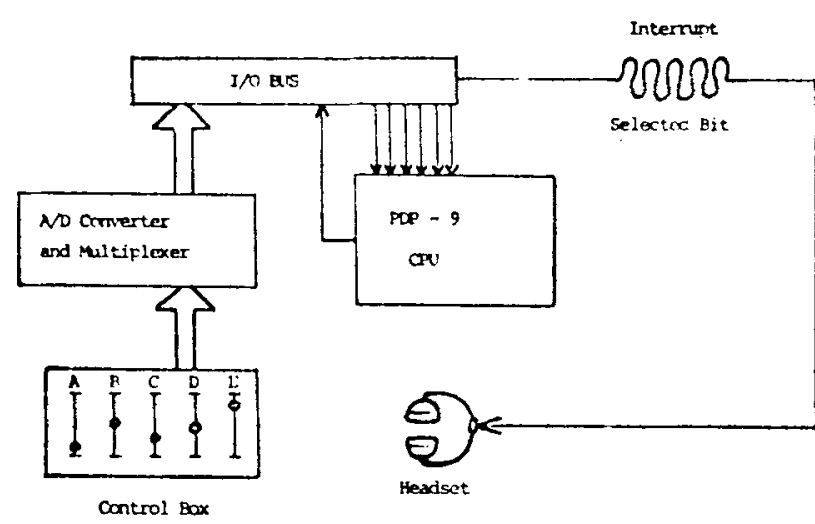

Fig. 3. On-line configuration for partial differential feedback (PDF) training.

Each of the knobs is an extension of a linear potentiometer mounted in the chassis and connected to a Model 138E A/D converter and a Model 139E multiplexer to a PDP.9 computer. The digitized information is then appropriately represented to the CPU as the set of values of the dimension, $X$. The software is written to scan and convert potentiometer voltages and to apply these to the function $\mathrm{F}(\mathrm{X}, \theta)$. The computed value. $\hat{Y}$, is linearly ${ }^{2}$ transformed to a bit frequency in the audio range on an $\mathrm{I} / \mathrm{O}$ interrupt line. This line can be any existing interrupt line which can be driven at rates up to $5 \mathrm{kc}$ for tone generation in the range $100-5,000 \mathrm{~Hz}$. The resulting signal is amplified and fed to a headset located near the stimulus display. The configuration is shown in Fig. 3.

\section{TONE GENERATION WITHOUT D/A CONVERTER}

Figure 4 illustrates the software logic required to generate the tone corresponding to any current potentiometer setting.

In the box labeled $\mathrm{A}$, the potentiomters are read and digitized to values of $X_{i j}$. The function is then evaluated, and $Y_{i}$ is stored as the current value. The tone is generated by using the current value of $Y_{i}$ to initialize a software counter and by activating an interrupt each time the counter is decremented to zero. This process is repeated for $90 \mathrm{msec}$, following which the potentiometers are sampled again.

If the potentiometers have not changed by an amount $\Delta \mathrm{X}_{\mathrm{ij}}$ in the 90 -msec interval, tone generation continues with an approximate 500-microsec lapse. If one of the potentiometers has changed. a new value for $Y_{i}$ is computed. Computation will require $3.5 \mathrm{msec}$ out of a $90-\mathrm{msec}$ tone, or perhaps $10 \mathrm{msec}$ as a worst case.

The entire software package consumes less than $4 \mathrm{~K}$ of core. If a $\mathrm{D} / \mathrm{A}$ converter is available. the potentiometers are sampled, $Y_{i}$ is calculated. and the $D / A$ converter is loaded with a number which will produce the required tone. Tone generation is completely overlapped with potentiometer sampling and with calculation of $Y_{i}$. This method produces more continuous frequency changes and simplifies the programming effort.

The task is arranged so as to place the stimulus configuration under $S$ control. The $S$ manipulates the values of the "attributes" by sliding the knobs up or down, and obtains virtually instant outcome feedback by attending to the changing tonal frequency being continuously fed to the headset. It is not necessary to anchor the task for the $\mathrm{S}$, but this may be accomplished by indicating to him that elevated positions of the knobs imply "more" of the attribute than do lowered positions, or vice versa, and that elevated tones from the speaker signity that more extreme values of the criterion dimension are to be associated with the stimulus configuration. On the other hand, the $S$ can determine these facts readily through trial-and-error performance at the control box.

Were the $\mathrm{E}$ to rearrange the knobs into completely different configurations at each moment, then have the $S$ "guess" at the value of the criterion, and then feed back to $S$ a measure of the true value in the form of a tonal frequency, he would be providing training in a classical SRO mode. In fact, the PDF task is conceptually different, and radically so. First, the stimulus is under control of S, not E. Second, outcome feedback does not follow S's response, but is present automatically without the necessity of a response at all. Third, the requirement for an observable response during acquisition is abandoned. The only observable response in this paradigm is S's activity at the control box, an activity consisting exclusively of a rearrangement of the stimulus.

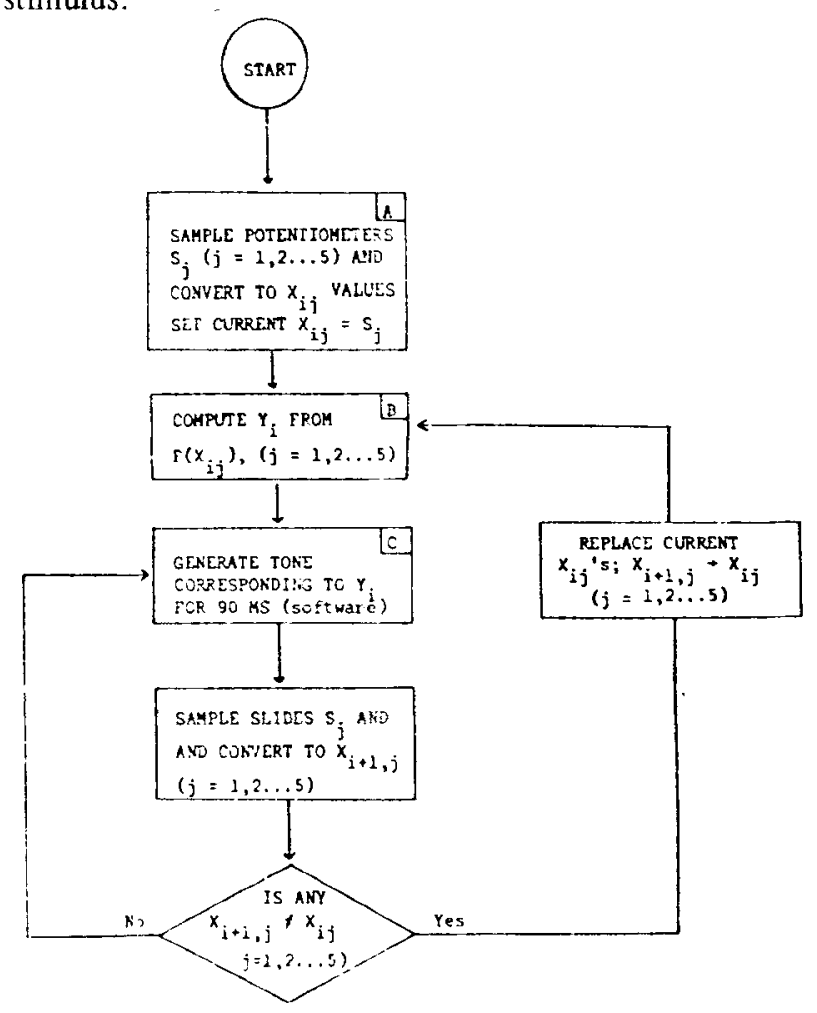

Fig. 4. Tone generation without $D / A$ equipment. 
Fourth, temporal or order effects which, classically, Es go to great pains to neutralize or balance out through randomization, are instead emphasized in the continuously changing nature of the stimulus.

Finally, the structure of the task is unique in the manner in which stimulus information is associated with the essential properties of the desired performance. Since it is $\mathbf{S}$ who alters the cues, he thereby directly effects changes in outcome. There are both visual and proprioceptive correlates of S's performance as he adjusts the cue values, and these are directly associated with perceived changes in pitch.

To clarify the meaning of the term partial differential feedback, let the stimulus attributes and the response dimension be the coordinate axes of an $s+1$ dimensional hyperspace such that, at any instant, the momentary values of the attributes define a value on the response dimension. Changes in the values of any of the attributes produce a change in the value of the response dimension at the given point in the s-dimensional subspace. The amount of change is given precisely by $\partial \hat{Y} / \partial X$, where $\hat{Y}$ is defined as in Eq. 1 .

\section{REFERENCES}

Castellan, N. J., Jr. The effect of different types of feedback in multiple-cue probability learning. Oregon Research Institute Research Bulletin, 1972, 12, No. 7 .

Hammond, K. R., \& Boyle, P. J. R. Quasi-rationality, quarrels, and new conceptions of feedback. Program on Cognitive Processes Report No. 130, Institute of Behavioral Science, University of Colorado, 1971.

Uhl, C. N, An analysis of dimensional judgment concepts. Unpublished doctoral dissertation, University of Oregon, 1960.

\section{NOTES}

1. Hoffman, P. J. Partial differential feedback: A paradigm for the acquisition of multidimensional concepts. Submitted for publication.

2. A log or power function might well be considered, so as to transform the range of output frequencies to an equal-appearing-interval scale of pitch. This is easily added to the software package without appreciable effects on timing. 